\title{
SARS-CoV-2 serology increases diagnostic accuracy in CT-suspected, PCR-negative COVID-19 patients during pandemic
}

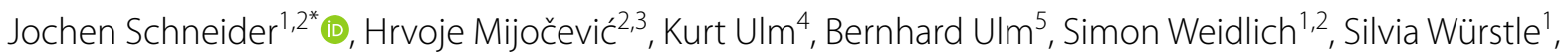
Kathrin Rothe ${ }^{2,6}$, Matthias Treiber ${ }^{1}$, Roman lakoubov ${ }^{1}$, Ulrich Mayr ${ }^{1}$, Tobias Lahmer ${ }^{1}$, Sebastian Rasch', Alexander Herner ${ }^{1}$, Egon Burian ${ }^{7}$, Fabian Lohöfer ${ }^{7}$, Rickmer Braren ${ }^{7}$, Marcus R. Makowski ${ }^{7}$, Roland M. Schmid ${ }^{1}$, Ulrike Protzer ${ }^{2,3}$, Christoph Spinner ${ }^{1,2+}$ and Fabian Geisler ${ }^{*^{*}+}$

\begin{abstract}
Background: In the absence of PCR detection of SARS-CoV-2 RNA, accurate diagnosis of COVID-19 is challenging. Low-dose computed tomography (CT) detects pulmonary infiltrates with high sensitivity, but findings may be nonspecific. This study assesses the diagnostic value of SARS-CoV-2 serology for patients with distinct CT features but negative PCR.
\end{abstract}

Methods: IgM/lgG chemiluminescent immunoassay was performed for 107 patients with confirmed (group A: PCR $+; C T \pm$ ) and 46 patients with suspected (group B: repetitive PCR-; CT +) COVID-19, admitted to a German university hospital during the pandemic's first wave. A standardized, in-house CT classification of radiological signs of a viral pneumonia was used to assess the probability of COVID-19.

Results: Seroconversion rates (SR) determined on day 5, 10, 15, 20 and 25 after symptom onset (SO) were 8\%, 25\%, $65 \%, 76 \%$ and $91 \%$ for group A, and $0 \%, 10 \%, 19 \%, 37 \%$ and $46 \%$ for group B, respectively; $(p<0.01)$. Compared to hospitalized patients with a non-complicated course (non-ICU patients), seroconversion tended to occur at lower frequency and delayed in patients on intensive care units. SR of patients with CT findings classified as high certainty for COVID-19 were $8 \%, 22 \%, 68 \%, 79 \%$ and $93 \%$ in group A, compared with $0 \%, 15 \%, 28 \%, 50 \%$ and $50 \%$ in group B $(p<0.01)$. SARS-CoV-2 serology established a definite diagnosis in 12/46 group B patients. In 88\% (8/9) of patients with negative serology $>14$ days after symptom onset (group B), clinico-radiological consensus reassessment revealed probable diagnoses other than COVID-19. Sensitivity of SARS-CoV-2 serology was superior to PCR $>17 \mathrm{~d}$ after symptom onset.

Conclusions: Approximately one-third of patients with distinct COVID-19 CT findings are tested negative for SARSCoV-2 RNA by PCR rendering correct diagnosis difficult. Implementation of SARS-CoV-2 serology testing alongside current CT/PCR-based diagnostic algorithms improves discrimination between COVID-19-related and non-related pulmonary infiltrates in PCR negative patients. However, sensitivity of SARS-CoV-2 serology strongly depends on the time of testing and becomes superior to PCR after the $2^{\text {nd }}$ week following symptom onset.

\footnotetext{
*Correspondence: Jochen.Schneider@tum.de; Fabian.geisler@mri.tum.de

${ }^{\dagger}$ Christoph Spinner and Fabian Geisler contributed equally to this work

1 Department of Internal Medicine II, School of Medicine, Technical

University of Munich, Munich, Germany

Full list of author information is available at the end of the article
}

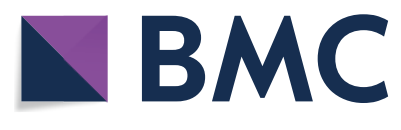

The Author(s) 2021. Open Access This article is licensed under a Creative Commons Attribution 4.0 International License, which permits use, sharing, adaptation, distribution and reproduction in any medium or format, as long as you give appropriate credit to the original author(s) and the source, provide a link to the Creative Commons licence, and indicate if changes were made. The images or other third party material in this article are included in the article's Creative Commons licence, unless indicated otherwise in a credit line to the material. If material is not included in the article's Creative Commons licence and your intended use is not permitted by statutory regulation or exceeds the permitted use, you will need to obtain permission directly from the copyright holder. To view a copy of this licence, visit http://creativecommons.org/licenses/by/4.0/. The Creative Commons Public Domain Dedication waiver (http://creativeco mmons.org/publicdomain/zero/1.0/) applies to the data made available in this article, unless otherwise stated in a credit line to the data. 
Keywords: COVID-19, SARS-CoV-2, Serology, Computed tomography, Efficacy, Accuracy

\section{Background}

To maintain functional patient care during the Severe Acute Respiratory Syndrome Coronavirus 2 (SARSCoV-2) pandemic [1], it is crucial to protect healthcare facilities from nosocomial coronavirus disease 2019 (COVID-19) outbreaks. As such, every hospital needs to separate patients with and without COVID-19 via a reliable triage algorithm to minimize the risk of nosocomial transmission during a time of ever-increasing patient numbers. At our German university hospital, patients with suspected COVID-19 have been triaged using a modified low-dose, computed tomography (CT)-based algorithm, according to Zhang et al. [2]. Due to its rapid action and high detection sensitivity for viral pneumonia, CT has proved highly effective for identifying the majority of COVID-19 cases with a high pre-test probability during the peak of the COVID-19 pandemic [3]. However, a significant limitation of CT imaging is the limited specificity of the imaging findings. Thus, diagnostic uncertainty remains in cases of negative polymerase chain reaction (PCR) testing for SARS-CoV-2. Direct detection of the virus by PCR is only possible for a limited time span and thus may be less sensitive than CT [4]. Thus, in advanced disease stages of COVID-19 PCR may miss a correct diagnosis [5-8]. Moreover, pre-analytics such as the quality of nasopharyngeal swaps also have a significant impact on the sensitivity of PCR assays [9]. Consequently, approximately one-third of patients with distinct COVID-19 CT findings are tested negative for SARS-CoV-2 RNA by PCR rendering correct diagnosis difficult [4].

Infection control management of patients with suspected COVID-19 that cannot be confirmed later on is complex and cost-intensive. To reduce the risk of nosocomial transmission of COVID-19, these patients must be isolated from others and are treated with high personal protection equipment. To correctly diagnose COVID-19 patients, innovative solutions are required.

The main focus of this retrospective study was to evaluate SARS-CoV-2 serology as a supplementary diagnostic method to increase diagnostic accuracy for patients with suspected COVID-19.

\section{Methods}

\section{Study population}

Retrospective evaluation for inclusion in this study was performed for 183 patients with suspected or confirmed COVID-19 admitted to the University Hospital Klinikum rechts der Isar of the Technical University of Munich, or identified during their hospital stay, from the $4^{\text {th }}$ of March to $22^{\text {nd }}$ of April 2020 during the first wave of the COVID19 pandemic. All patients fulfilled at least one criterion, according to the Wuhan Triage Algorithm (respiratory symptoms/chills plus dyspnea/hypoxia and/or temperature $>37.3{ }^{\circ} \mathrm{C}$ and/or absolute lymphocyte counts $<1100 /$ $\mu \mathrm{L})$ [2], and therefore underwent both low-dose chest CT scanning and SARS-CoV-2 PCR testing of nasopharyngeal swab. Of 183 patients, 30 were excluded: one patient received immunoglobulin therapy due to secondary antibody insufficiency syndrome prior to hospitalization, five patients had incomplete data, and three patients had both negative $\mathrm{CT}$ and repeatedly negative PCR testing. As SARS-CoV-2 serology testing had not been implemented into clinical routine at our hospital from the very beginning of the COVID-19 pandemic, 21 patients without SARS-CoV-2 serology test results were excluded. Influenza and respiratory syncytial virus (RSV) co-infection were excluded in all patients using PCR (Cepheid GeneXpert RSV/FLU, California, USA). Patients with positive SARS-CoV-2 PCR \pm distinct COVID-19 CT features were defined as confirmed COVID-19 cases (group A), whereas patients with positive CT findings but negative PCR were classified as suspected cases (group B). Every patient underwent a standardized medical history assessment including the documentation of symptom onset prior to hospitalization. If the assessment could not be performed due to cognitive impairment, such as dementia or critical illness (9 patients), the day of hospital admission was defined as the date of symptom onset. Twelve patients first developed clinical symptoms during pre-existing hospitalization.

\section{Inclusion and exclusion criteria}

The following inclusion criteria were defined:

Age $\geq 17$

Availability of (low-dose) chest CT

SARS-CoV-2 PCR testing

Patients were excluded from the study in the following cases:

Plasma separation therapy or immunoglobulin substitution therapy before serological testing

Negative SARS-CoV-2 PCR and chest CT without COVID-19 suspicious findings

No SARS-CoV-2 serology available 
Incomplete medical records in terms of the abovelisted data criteria

\section{Ethics statement}

The study was approved by the Institutional Ethics Committee of the Klinikum rechts der Isar, Technical University of Munich, which operates according to the Declaration of Helsinki (Approval No. 247/20 S). Due to the retrospective study design, the need for written consent was waived.

\section{Sample collection}

In patients with suspected COVID-19, nasopharyngeal swab samples were collected according to a standardized protocol. On ICU, trachea-bronchial aspirates were alternatively obtained for SARS-COV-2 PCR. To protect our medical staff from COVID-19 transmission, all aerosol producing interventions like bronchoalveolar lavage were avoided if not absolute necessary from a clinical point of view. To economize laboratory capacity and testing efficiency during the pandemic situation, nasopharyngeal and throat swab specimens were pooled. In order to guarantee optimal detection of SARS-CoV-2 by PCR, a standardized swab collection and transport system (Sigma Virocult@ \& Transwab@ Sigma-Aldrich, St. Louis, Missouri, USA) complying with the CLSI standard M40-A for Quality Control of Specimen Transport Devices was used. Samples were stored at $4{ }^{\circ} \mathrm{C}$ until processed. For serological testing, $5-8 \mathrm{~mL}$ of blood serum was obtained.

\section{Laboratory testing}

PCR and serological processing were performed at a clinical virology laboratory (Institute of Virology, Technical University of Munich), accredited according to DIN EN ISO 15,189. Ribonucleic acid (RNA) was extracted using the mSample Preparation System DNA kit with standard protocol for the simultaneous extraction of DNA and RNA on a m2000sp device (Abbott, Wiesbaden, Germany). SARS-CoV-2 PCR was performed using "in-house" real-time PCRs on a TaqMan device and primer and probe sets targeting the SARSCoV-2 N gene according to the protocol of the Center for Disease Control and Prevention (CDC), Atlanta, USA. For screening and quantification the N1 and for confirmation the N3 primer/probe sets were used.

\section{Validation of SARS-CoV-2 serology}

Detection of serum IgM and IgG antibodies for SARSCoV-2 was performed using a paramagnetic particle chemiluminescent immunoassay (CLIA) on an iFlash 1800 immunoassay analyzer (Shenzhen Yhlo Biotech Co., Shenzhen, China). Specificity of SARS-CoV-2 IgM and IgG serology was validated in-house by analyzing 84 control sera, which were collected during 2019 and stored at $-20{ }^{\circ} \mathrm{C}$ in our biobank for research purposes. Of 84 control sera, two were positive for SARS-CoV-2 IgG and one for IgM, resulting in an overall specificity of $98 \%$. According to the manufacturer's specification, sensitivity and specificity of the kits are $97.3 \%$ and $96 \%$ for IgG, and $86.1 \%$ and $99.2 \%$ for IgM. In a recently published study [10], sensitivity of $97.6 \%$ for IgG and $87.8 \%$ for IgM was observed, with an overall specificity of $100 \%$.

\section{Computed tomography}

Study patients underwent a low-dose chest CT using a 256-row scanner (iCT, Philips Healthcare, Best, Netherlands). All CT scans were evaluated for distinct COVID-19 features by a 1st-4th year radiology resident and re-evaluated by an experienced attending radiologist (2-15 years of experience) during routine reporting. CT features were assessed using a standardized in-house classification with five levels of diagnostic certainty: $0=$ no signs of COVID-19 features; $1=$ infiltration or consolidation not typical for COVID-19 infection; $2=$ early stage COVID- 19 possible; $3=$ typical CT features compatible with early COVID-19; $4=$ typical CT features compatible with advanced COVID-19.

\section{Statistical analysis}

The distribution of continuous variables is described by median and range. Categorical data are presented as absolute and relative frequencies. Kaplan-Meier survival curves were chosen to illustrate the time from symptom onset to the event of seroconversion to SARS-CoV-2 serology. Comparison of Kaplan-Meier survival curves was conducted by log-rank testing. Statistical hypothesis testing was performed on two-sided exploratory $0.05^{*}$ significance levels. All analyses were conducted using IBM SPSS Statistics 20 (version 26, IBM Corp., Armonk, New York, USA) and R (version 3.4.3, R Foundation for Statistical Computing, Vienna, Austria).

\section{Results \\ Baseline characteristics}

In total, 153 patients met the criteria for inclusion. The median age of the study population was 68 years (range 
Table 1 Difference in baseline characteristics between group A and group B

\begin{tabular}{|c|c|c|c|}
\hline Baseline characteristics & $\begin{array}{l}\text { In total } \\
\text { Absolute value (\%) }\end{array}$ & $\begin{array}{l}\text { Group } A \\
\text { (PCR }+; \mathrm{CT} \pm \text { ) } \\
\text { Absolute value (\%) }\end{array}$ & $\begin{array}{l}\text { Group B } \\
\text { (PCR-; CT +) } \\
\text { Absolute value } \\
(\%)\end{array}$ \\
\hline Number of patients & $153(100)$ & $107(70)$ & $46(30)$ \\
\hline Age (median) & 68 & 67 & 69 \\
\hline Sex (male/female) & $95 / 58$ & $70 / 37$ & $25 / 21$ \\
\hline \multicolumn{4}{|l|}{ Comorbidities (Comorbidity Score index points*) } \\
\hline Coronary artery disease $\mathrm{a}^{\mathrm{a}}(1$ point $)$ & $17(11)$ & $15(14)$ & $2(4)$ \\
\hline Heart disease $^{\mathrm{b}}$ (1 point) & $41(27)$ & $27(25)$ & $14(30)$ \\
\hline Cerebrovascular disease ${ }^{c}$ (1 point) & $11(7)$ & $6(5)$ & $5(11)$ \\
\hline Dementia/Parkinson disease (1 point) & $17(11)$ & $8(8)$ & $9(20)$ \\
\hline Gastric ulcer disease (1 point) & $5(3)$ & $4(4)$ & $1(2)$ \\
\hline Chronic pulmonary disease (1 point) & $17(11)$ & $12(11)$ & $5(11)$ \\
\hline Peripheral vascular disease (1 point) & $9(6)$ & $7(7)$ & $2(4)$ \\
\hline Mild liver disease ${ }^{d}$ (1 point) & $1(1)$ & $1(1)$ & $0(0)$ \\
\hline Liver cirrhosis (3 point) & $4(3)$ & $3(3)$ & $1(2)$ \\
\hline Diabetes without end organ failure (1 point) & $22(14)$ & $18(17)$ & $4(9)$ \\
\hline Diabetes with end organ failure (2 points) & $8(5)$ & $7(7)$ & $1(2)$ \\
\hline Renal insufficiency (2 points) & $14(9)$ & $11(10)$ & $3(7)$ \\
\hline Active tumor disease $\mathrm{e}^{\mathrm{e}}(2$ points) & $11(7)$ & $9(8)$ & $2(4)$ \\
\hline Metastatic tumor disease (6 points) & $16(10)$ & $8(8)$ & $8(17)$ \\
\hline AIDS (6 points) & - & - & - \\
\hline Comorbidity Score points in total (median/mean) & $314(1 / 2.05)$ & $209(1 / 1.95)$ & $105(1 / 2.28)$ \\
\hline Median duration of hospitalization in days & 16 & 16 & 13.5 \\
\hline $\begin{array}{l}\text { Number of patients with typical CT findings compatible with a } \\
\text { high level of certainty for COVID-19 }\end{array}$ & $125(82)$ & $85(79)$ & $30(65)$ \\
\hline Deaths & $25(16)$ & $18(17)$ & $7(15)$ \\
\hline ICU admission & $53(35)$ & $41(38)$ & $12(26)$ \\
\hline Treatment (Remdesivir) & 17 & 17 & 0 \\
\hline
\end{tabular}

*Modified comorbidity index referring to Charlson et al. [11].

${ }^{a}$ Including stenting or aortocoronary bypass; ${ }^{b}$ including arterial fibrillation, congestive heart failure; ${ }^{c}$ including transient ischemic attack (TIA), stroke; ${ }^{d}$ defined

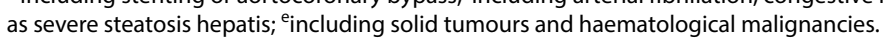

Table 2 Level of certainty for COVID-19 based on CT findings using in-house radiology classification

\begin{tabular}{llll}
\hline $\begin{array}{l}\text { Level of certainty for } \\
\text { COVID-19 }\end{array}$ & COVID-19 CT classification & $\begin{array}{l}\text { Group A } \\
(\mathbf{n = 1 0 7 )} \\
\text { abs. no (\%) }\end{array}$ & $\begin{array}{l}\text { Group B } \\
\mathbf{( n = 4 6 )} \\
\text { abs. no } \\
\mathbf{( \% )}\end{array}$ \\
\hline Low & & $4(4)$ & - \\
Low & No signs of COVID-19 CT features (category 0) & - & $4(2)$ \\
Low & Infiltration or consolidation not typical for COVID-19 (category 1) & $17(18)$ & $30(14)$ \\
High & Early stage of COVID-19 infection possible (category 2) & $23(25)$ & $20(9)$ \\
High & Typical CT features compatible with early COVID-19 (category 3) & $56(60)$ & $46(21)$ \\
\hline
\end{tabular}

17-100). Of the 153 patients, 58 (38\%) were female. Median time from symptom onset to hospital admission was 6 days (range $0-32$ ). Of the 153 patients, 53 (35\%) required transfer to the intensive care unit (ICU) during hospitalization. In-house mortality was $16 \%(25 / 153$ patients) at the time of analysis (22 $2^{\text {th }}$ May 2020). According to the study criteria, 107 patients (107/153; 70\%) were defined as confirmed COVID-19 (group A: PCR+, 


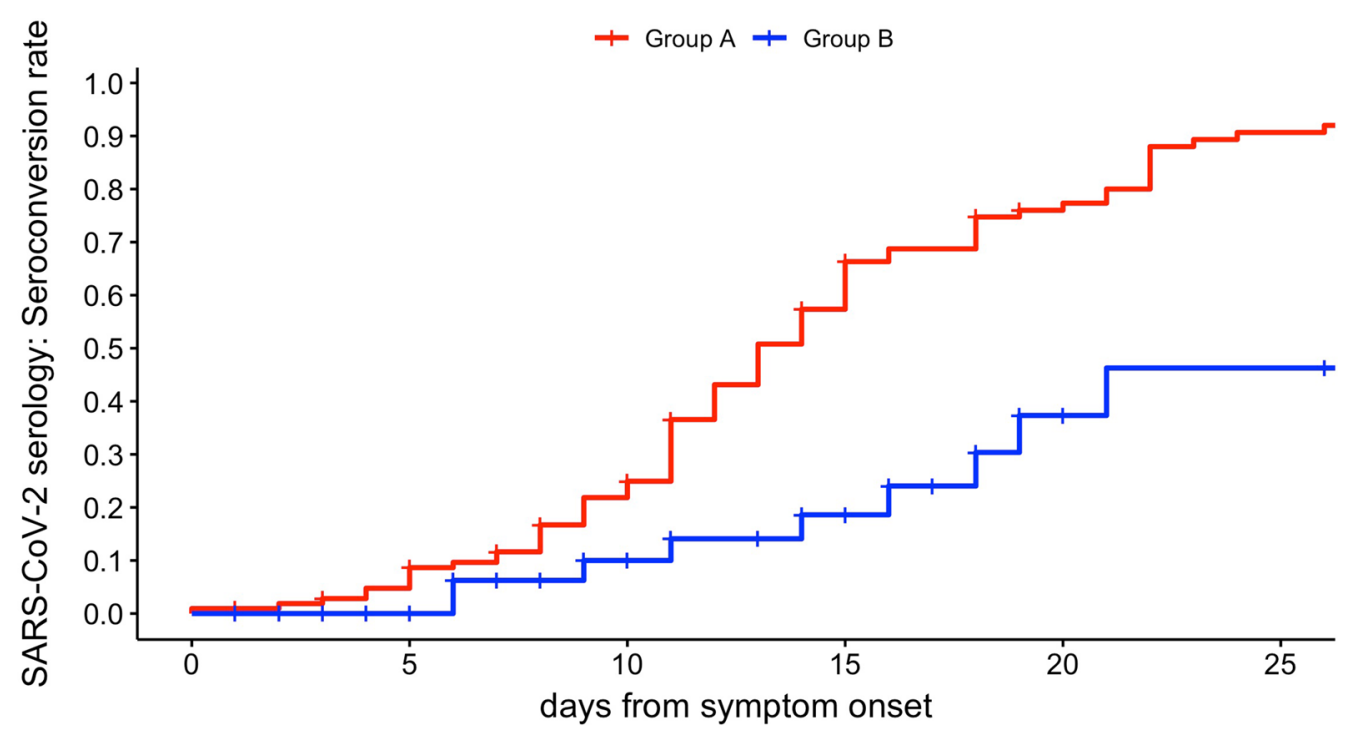

Number at risk

\begin{tabular}{|c|c|c|c|c|c|c|}
\hline Group A - & 107 & 98 & 76 & 38 & 18 & 7 \\
\hline Group B & 46 & 37 & 23 & 16 & 8 & 6 \\
\hline & 0 & 5 & 10 & 15 & 20 & 25 \\
\hline
\end{tabular}

Fig. 1 Illustration of seroconversion rates following SO in patients with confirmed COVID-19 (group A, solid red line) and patients with suspected COVID-19 (group B, solid blue line); p-value $<0.01$

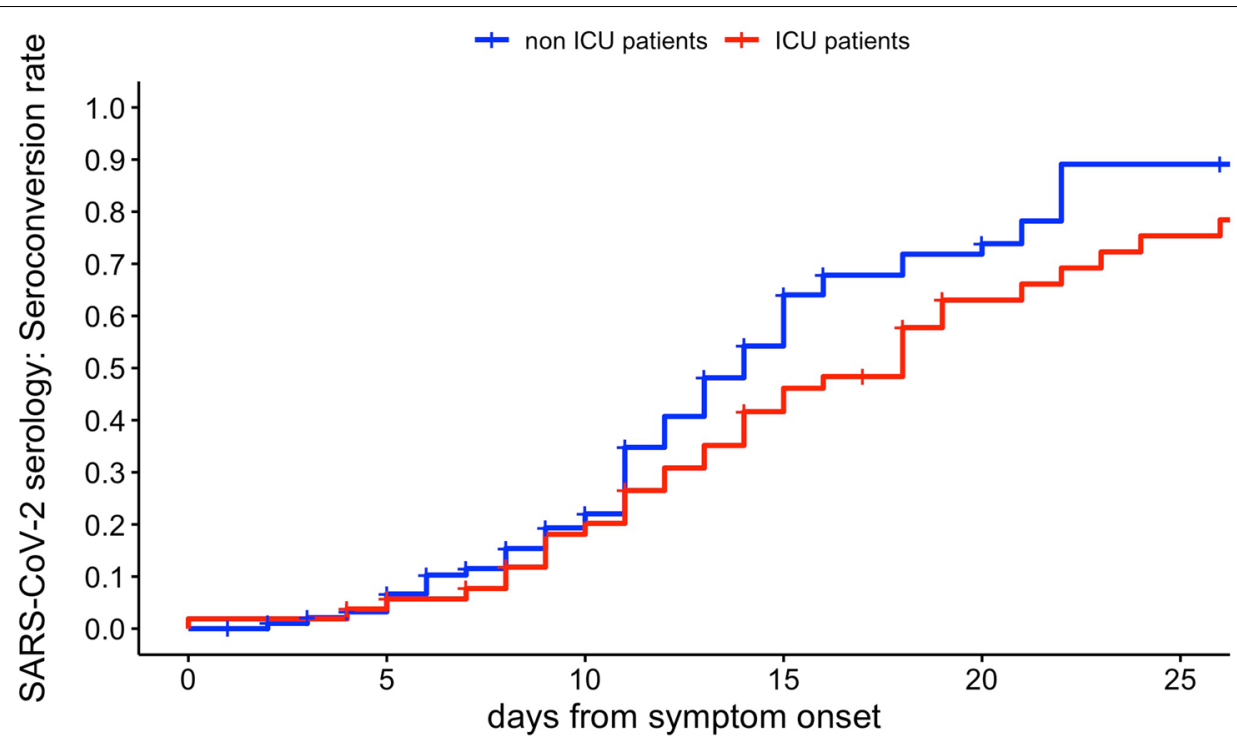

Number at risk

\begin{tabular}{r|cccccc} 
non ICU patients - & 100 & 85 & 60 & 28 & 14 & 5 \\
ICU patients - & 53 & 50 & 39 & 26 & 12 & 8 \\
\hline 0 & 5 & days from symptom onset & & 20 & 25
\end{tabular}

Fig. 2 Comparison of seroconversion rates over time between ICU patients (solid red line) and non-ICU patients (solid blue line); $p=0.11$ 
$\mathrm{CT} \pm)$ and 46 patients $(46 / 153 ; 30 \%)$ as suspected COVID-19 (group B: PCR-, CT +). Table 1 shows the difference in baseline characteristics between patient group A and patient group B. Table 2 illustrates the distribution of $\mathrm{CT}$ findings between group A and group B according to the level of certainty for COVID-19 using the in-house CT-based COVID-19 classification.

\section{SARS-CoV-2 serology: results}

99 of the $153(65 \%)$ patients were SARS-CoV-2 seropositive. Of the seropositive patients, 77\% (76/99) showed both IgM and IgG positivity, while 23\% (23/99) of patients were only IgG positive. IgM and IgG seroconversions occurred in median 14 days (range 4-32) and 13 days (range 2-32) following SO, respectively.

Figure 1 displays the difference in seroconversion rates of SARS-CoV-2 serology distinctly for confirmed (group A) and suspected COVID-19 cases (group B). On days 5, $10,15,20$, and 25 following SO, seroconversion rates of group A and group B were $8 \%, 25 \%, 65 \%, 76 \%, 91 \%$, and $0 \%, 10 \%, 19 \%, 37 \%$ and $46 \%(\mathrm{p}<0.01)$, respectively.

In terms of severity of COVID-19 (Fig. 2), seroconversion was higher and occurred earlier in non-ICU patients than in ICU patients. At 5, 10, 15, 20 and 25 days following $\mathrm{SO}$, seroconversion was $8 \%, 22 \%, 64 \%, 74 \%$, and $89 \%$,

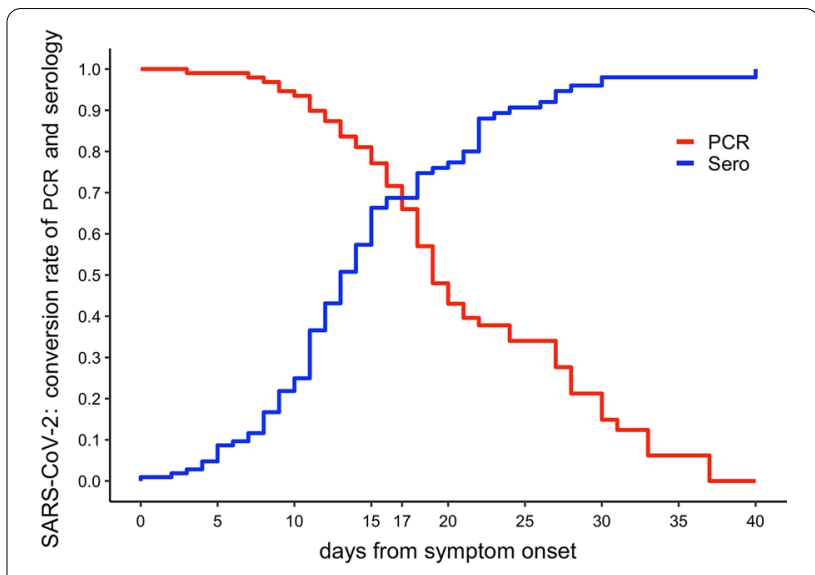

Fig. 4 Analysis of conversion rate of SARS-CoV-2 serology and SARS-COV-2 PCR test during hospitalization

respectively, in non-ICU patients, and 6\%, 20\%, 46\%, 63\%, $75 \%$, respectively, in ICU patients $(\mathrm{p}=0.11)$.

The impact of CT findings in relation to likelihood of COVID-19 based on seroconversion is demonstrated in Fig. 3. Seroconversion rates of PCR-positive patients (group A) with CT findings compatible with a high level of certainty for COVID-19 (including only category 3 and

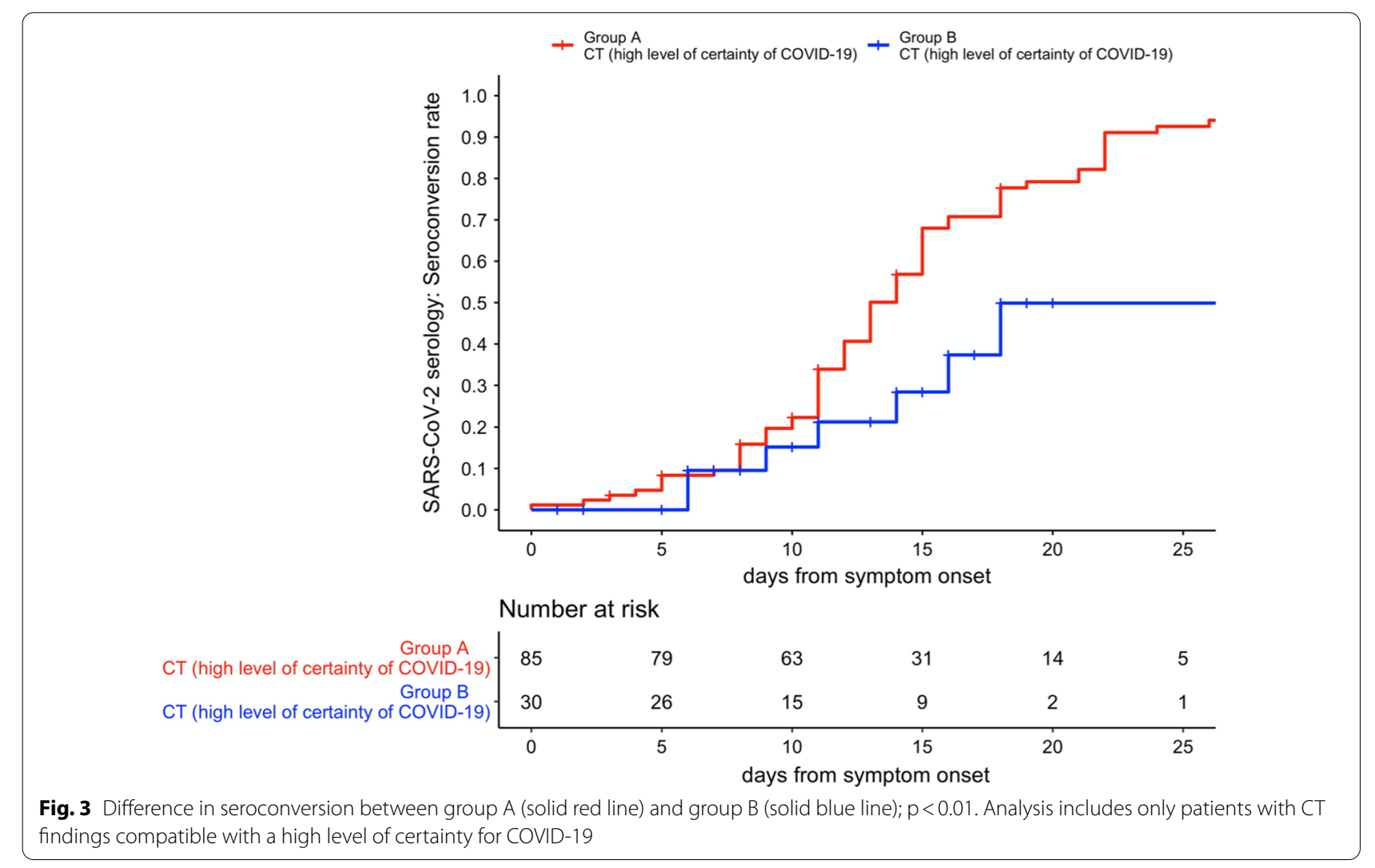


category 4 according to the in-house CT classification; Table 2 ) were $8 \%, 22 \%, 68 \%, 79 \%$, and $93 \%$ after $5,10,15$, 20 and 25 days, respectively, following SO. In contrast, seroconversion rates of PCR-negative patients (group B) with CT findings consistent with high level of certainty for COVID-19 (including only category 3 and category 4 according to the in-house CT classification; Table 2) were $0 \%, 15 \%, 28 \%, 50 \%$, and $50 \%$ at the same time intervals following SO $(\mathrm{p}<0.01)$.

Seroconversion and PCR conversion (defined as time point of negativeSARS-CoV-2 PCR) were analyzed during hospitalization (Fig. 4). In the early phase of infection, the detection efficiency of PCR for COVID-19 was higher compared with SARS-CoV-2 serology, whereas detection efficiency of serology for COVID-19 became superior to PCR $>17$ days following symptom onset.

In 12 of $46(26 \%)$ suspected cases (group B), positive SARS-CoV-2 serology provided definitive diagnoses of COVID-19. All of these patients underwent at least two SARS-CoV-2 PCR tests (range 2-6). Eight of these 12 patients had typical CT features compatible with a high level of certainty for COVID-19 according to our COVID-19 radiological classification. Based on the findings by Wölfel et al., [6] which showed a COVID19 seroconversion rate of $100 \%$ within 14 days after SO, patients from group A (confirmed COVID-19) and group B (suspected COVID-19) with at least one late serological test $>14$ days were separately analyzed in subgroup A1 and subgroup B1. Seroconversion rates of subgroup A1 (confirmed COVID-19) and subgroup B1 (suspected COVID-19) were $92 \%$ (59/64 patients) versus 52\% (10/19 patients), resulting in a difference of $40 \%$. In subgroup A1, seroconversion did not occur in $8 \%(5 / 64)$ of patients; all five patients suffered from immunosuppressive diseases such as active neoplasia (four patients) or diabetes mellitus (one patient). In comparison, 48\% (9/19) of suspected cases with at least one SARS-CoV-2 serology test > 14 days after SO (subgroup B1) had no seroconversion. Retrospective interdisciplinary clinico-radiolocial consensus re-evaluation of these nine patients revealed possible causes other than COVID-19 for their CT findings: one patient underwent bronchoalveolar lavage prior to CT scanning and developed lung edema due to iatrogenic fluid instillation; three patients showed signs of focal lung edema due to cardiac failure; one patient had pulmonary metastatic breast cancer with presumed lymphangiosis carcinomatosis, mimicking COVID-19; one patient's CT showed signs of breathing artifacts; two patients had CT features in retrospect rather compatible with bacterial pneumonia (lobar pneumonia surrounding by ground-glass opacities with a parapneumonic pleural effusion) as a possible differential diagnosis. For the final one of these nine patients, no other rational differential diagnosis besides COVID-19 seemed to be reasonable.

\section{Discussion}

The CT-based "Wuhan Triage Algorithm" was developed during the peak of the COVID-19 pandemic in Wuhan, China [2]. A core element of this triage algorithm is a low-dose chest-CT, providing faster and more sensitive identification of patients with COVID-19 compared to detection of SARS-CoV-2 RNA by RT-PCR. However, the positive predictive value of highly sensitive diagnostic methods depends heavily on the prevalence of the suspected disease in the tested cohort. In many countries all over the world, nationwide lock-downs and social distancing measures have flattened the curve of the COVID19 pandemic [12], and consequently the proportion of COVID-19 in patients attending the Emergency Department with fever or respiratory symptoms decreased. This may result in an increase in the numbers of false-positive CT findings for COVID-19. Physicians faced a similar problem regarding diagnosis of other infections such as Clostridium difficile or human immunodeficiency virus (HIV) - highly sensitive tests with low positive predictive values due to low prevalence. This problem can be solved by introducing a two-step diagnostic algorithm based on a highly sensitive screening test, followed by a confirmatory test [13]. In this study, SARS-CoV-2 serology was evaluated as a confirmatory test for patients with CT findings suspect of COVID-19. Out of 153 patients, $46(30 \%)$ had a suspected diagnosis of COVID-19, which was based on a combination of clinical findings and $\mathrm{CT}$ imaging results. Tao $\mathrm{Ai}$ et al. [4] analyzed the correlation of chest CT and PCR testing in COVID-19 in China, revealing a similar proportion of suspected COVID-19 cases with negative PCR but positive CT findings. For the patients in our study with repetitive negative PCR and positive CT findings (group B), positive serology led to the final diagnosis of COVID-19 in 26\% (12/46) of cases. As illustrated in the Methods section, the specificity of SARS-CoV-2 serology was found to be high (98\%). Nevertheless, differentiation between acute infection and postinfection IgG responses is challenging in the absence of any IgM response. In the current study, in 23\% (23/99) of seroconverted patients only IgG was detected. As an increasing seroprevalence of COVID-19 in the population can be expected in the months following the pandemic, SARS-CoV-2 serology may mislead clinicians to diagnose acute COVID-19 in their patients. Therefore, to increase pre-test probability, SARS-CoV-2 serology could be embedded into a two-step diagnostic algorithm using $\mathrm{CT}$ as a screening test, followed by SARS-CoV-2 serology and PCR as a confirmation test when acute infection is suspected. This way, a diagnosis of COVID-19 can 
be confirmed despite repetitive negative PCR testing, if patients present positive SARS-CoV-2 serology and show distinct COVID-19 CT findings.

The sensitivity of SARS-CoV-2 serology increased with time since symptom onset. In PCR-positive patients (group A), SARS-CoV-2 serology achieved a sensitivity of less than $15 \%$ within the first week and up to $92 \%$ after the third week following SO. Wölfel et al. [6] analyzed seroconversion of patients suffering from COVID19 based on an assay using cloned spike protein of SARS-COVID-19. In early sera, collected between days three and six, no seroconversion was observed; however, all patients developed an antibody response during monitoring for at least two weeks. These findings clearly illustrate that the use of SARS-CoV-2 serology as a tool to diagnose COVID-19 in the early stage of infection is limited.

In the current study, the median time from symptom onset to hospital admission was six days, which is comparable to other clinical trials $[10,14]$. Less than $15 \%$ of confirmed cases had a positive antibody response on day six following SO, as shown in Fig. 1 . In the early phase of infection ( $\leq 17$ days), the sensitivity of PCR was superior to SARS-CoV-2 serology. However, with prolonged duration of symptoms ( $>17$ days), PCR became inferior to the detection efficiency of SARS-CoV-2 serology. In comparison with the data from Wölfel et al. observing a seroconversion of all patients within 14 days after symptom onset [6], seroconversion did not occur in all patients and was delayed in the current study, which could be related to the high proportion of severely ill patients. Wölfel et al. [6] analyzed antibody response against COVID-19 in nonseverely ill patients during the early containment phase of the COVID-19 pandemic in Germany and, in contrast, $35 \%$ of our study patients had to be transferred to the intensive care unit. As shown in Fig. 2, humoral response against COVID-19 in this study tended to occur earlier and more frequently in non-ICU patients than in ICU patients. In contrast to the findings of our study, Fourati et al. [15] observed that all patients admitted to ICU had a positive serology SARS-CoV-2 upon admission. We believe that these discrepant findings can be explained by different test performances of SARS-CoV-2 assays due to the use different antigens. Fourati et al. [15] used an IgG/ IgA assay, whereas SARS-CoV-2 antibody detection was conducted by a IgG/IgM assay in the current study. Interestingly, Sun et al. [16] analyzed IgM and IgG responses against SARS-CoV-2 nucleocapsid $(\mathrm{N})$ and spike (S) protein after symptom onset depending on the severity of COVID-19. They found that ICU patients had higher $\mathrm{N}-\operatorname{IgG}$ than S-IgG than non-ICU patients. Furthermore, S-IgG increased slower than N-IgG in ICU patients after symptom onset. In line with the results of our study, $\mathrm{Qu}$ et al. [10] analysed the profile of IgG and IgM antibodies against severe acute respiratory syndrome coronavirus 2 (SARS-CoV-2). IgG and IgM antibodies against SARS$\mathrm{CoV}-2$ were measured using the same iFlash-SARSCoV-2 IgG/IgM chemiluminescent immunoassay kit (C86095G/ C86095M, YHLO BIOTECH, Shenzhen) as we did in our study. Compared to patients with mild or moderate COVID-19, they also observed delayed IgG and IgM antibody responses in critical ill patients suffering from COVID-19.

Interestingly, median seroconversion of IgG was observed slightly earlier than median seroconversion of IgM. This could be related to a well-known technical problem with serological assays: avidity of IgG antibodies is higher compared to that of IgM antibodies, and so IgG can outcompete IgM for viral epitopes. In addition, numbers are still low. Thus, this phenomenon requires further analysis.

The difference in seroconversion rates between PCRpositive and PCR-negative patients highlights the intermediate specificity of CT imaging findings for COVID19 pneumonia. At day 25 after symptom onset, seroconversion occurred only in up to $46 \%$ of PCR-negative patients, compared with $91 \%$ of PCR-positive patients (Fig. 1). Viral infections such as RSV, influenza, or human metapneumovirus [17] along with several non-infectious causes, can present similar CT changes such as multifocal consolidation or ground-glass opacities in the lungs. Diagnosis of COVID-19 in all group B patients with negative SARS-CoV-2 serology $>14 \mathrm{~d}$ after SO (9/19 patients) was retrospectively re-evaluated by an interdisciplinary Senior Consultant Physician Team from the Department of Radiology and the Department of Internal Medicine and Infectious Diseases. Only one patient out of nine had a differential diagnosis workup which revealed no other possible cause for the CT findings. Most of the remaining patients (4/9) suffered from pre-existing cardiovascular diseases and showed radiological signs of pulmonary edema such as septal lines or peribronchial cuffing. In contrast to characteristic lobular pneumonia, CT findings for COVID-19 are often diffuse and bilaterally localized, and therefore sometimes difficult to distinguish from a cardiogenic pulmonary edema. Pulmonary edema can also be caused by the underlying inflammatory process of pneumonia, which further complicates the distinction between COVID-19 and cardiogenic pulmonary edema. Clinical and radiological re-evaluation based on response to diuretic therapy may be the best way to differentiate a cardiogenic pulmonary lung edema from COVID-related CT findings. The CT of one patient with a pre-existing cardiovascular disease was initially rated as highly suspicious for COVID-19; however, seven days after the initiation of diuretic therapy, another $\mathrm{CT}$ scan 
was performed which no longer showed signs of typical COVID-19 related findings. From this experience it can be concluded that diagnosis of COVID-19 based solely on CT-findings is not highly reliable despite the high prevalence of COVID-19 in the current pandemic and therefore should either be confirmed by SARS-CoV-2 PCR or SARS-CoV-2 serology.

One limitation of this study is that the time points of serological testing were not standardized due to its retrospective design, which represents a potential bias to the time of seroconversion. Nevertheless, this potential bias does not appear to have impacted upon the major finding of the study, namely that a significant proportion of typical COVID-19 CT findings could be false positive.

\section{Conclusion}

Pre-test probability of SARS-CoV-2 serology in patients with typical COVID-19 CT findings can be considered sufficient to confirm COVID-19 despite the absence of a positive PCR. Nonetheless, negative SARS-CoV-2 serology does not exclude COVID-19. As the detection probability of antibody response increases with duration from symptom onset, the repetition of serological testing seems to be reasonable for PCR-negative patients who have recently developed symptoms. Finally, diagnosis of COVID-19 should be questioned in cases where patients have repetitive negative PCR and serological testing, despite distinct COVID-19 CT features.

\footnotetext{
Abbreviations

TIA: Transient ischemic attack; SR: Seroconversion rates; SO: Symptom onset; COVID-19: Coronavirus disease 2019; CT: Computed tomography; PCR: Polymerase chain reaction; d: Day; SARS-CoV-2: Severe Acute Respiratory Syndrome Coronavirus 2; RSV: Respiratory syncytial virus; CLIA: Chemiluminescent immunoassay.
}

\begin{abstract}
Acknowledgements
This study is dedicated to Professor Wolfgang Huber, who substantially contributed to this work and lost his fight for life during the COVID-19 pandemic Prof. Wolfgang Huber was a world-class expert in the field of hemodynamic monitoring on intensive care units. He was a major contributor to the clinical implementation of Pulse Contour Cardiac Output (PiCCO) technology, which has become indispensable in modern intensive care. During his time at the University Hospital Klinikum rechts der Isar, he trained physicians in intensive medical care with pleasant dedication and contributed widely to the publication of his research. As well as his outstanding knowledge in intensive care medicine, Prof. Wolfgang Huber had also substantial experience in the management of infectious diseases and gastroenterological conditions such as chronic inflammatory bowel disease, acute pancreatitis, and liver cirrhosis. With all due respect to you, Wolfgang: we will keep your legacy alive.
\end{abstract}

\section{Authors' contributions}

All authors agreed to be accountable for all aspects of the work in ensuring that questions related to the accuracy or integrity of any parts are appropriately investigated and resolved. All authors revised the manuscript critically for intellectual content and approved the final version for publication. JS: substantial contribution to conception and design of the study, and preparation and drafting of the article. HM.: substantial contribution to acquisition of data and methodology (validation of SARS-CoV-2 serology and provision of virology data). KU: substantial contribution to analysis and interpretation of data and drafting the article. $\mathrm{BH}$ : substantial contribution to analysis and interpretation of data and substantively revised the manuscript. SWe: substantial contribution to acquisition of data, input of raw data, data curation and substantively revised the manuscript. SWu: substantial contribution to analysis and interpretation of data acquisition of data and data curation. KR: substantial contribution to acquisition of data, data curation, and drafting the article. MT: substantial contribution to acquisition of data and input of raw data and validation of data. Rl: substantial contribution to acquisition of data, input of raw data and data curation. UM: substantial contribution to acquisition and input of data. TL: substantial contribution to acquisition and input of data as well as to conceptualization. AH: substantial contribution to acquisition of data and substantively revised the manuscript. SR: substantial contribution to acquisition and input of data. EB: substantial contribution to analysis and interpretation of data, including re-assessment of CT scans for COVID-19, and conceptualization regarding the establishment of COVID-19 radiological classification. FL: substantial contribution to analysis and interpretation of data, including re-assessment of CT scans for COVID-19), and drafting the article. RB: substantial contribution to analysis and interpretation of data, including re-assessment of CT scans for COVID-19, and conceptualization regarding the establishment of COVID-19 radiological classification. MM substantial contribution to conception and design, with oversight and leadership responsibility for the research. RS: substantial contribution to conception and design, with oversight and leadership responsibility for the research. UP: substantial contribution to conception and design, with oversight and leadership responsibility for the research. Substantial contribution to revision of the manuscript CS: substantial contribution to conception and design throughout evolution and realization of the study idea, and management and coordination responsibility for the research activity planning and execution. FG: substantial contribution to conception and design throughout evolution of overarching research goals and aims, management and coordination responsibility for the research activity planning and execution, and final approval of the manuscript. All authors read and approved the final manuscript.

\section{Funding}

Open Access funding enabled and organized by Projekt DEAL.

\section{Availability of data and materials}

The datasets used and/or analysed during the current study are available from the corresponding author on reasonable request.

\section{Declarations}

Ethics approval and consent to participate

Ethics Committee of Klinikum rechts der Isar, Technical University of Munich approved this study.

\section{Consent for publication}

Consent for publication has been obtained.

\section{Competing interests}

The authors declare that they have no competing interests.

\begin{abstract}
Author details
${ }^{1}$ Department of Internal Medicine II, School of Medicine, Technical University of Munich, Munich, Germany. ${ }^{2}$ German Center for Infection Research (DZIF), partner site Munich, Munich, Germany. ${ }^{3}$ Institute for Virology, School of Medicine, Technical University of Munich, Munich, Germany. ${ }^{4}$ Institute for Medical Statistics and Epidemiology, School of Medicine, Technical University of Munich, Munich, Germany. ${ }^{5}$ Department of Anaesthesiology and Intensive Care Medicine, School of Medicine, Technical University of Munich, Munich, Germany. ${ }^{6}$ Institute for Medical Microbiology, Immunology and Hygiene, School of Medicine, Technical University of Munich, Munich, Germany. ${ }^{7}$ Institute for Diagnostic and Interventional Radiology, School of Medicine, Technical University of Munich, Munich, Germany.
\end{abstract}

Received: 29 July 2020 Accepted: 14 April 2021

Published online: 23 April 2021 


\section{References}

1. Wee LE, Conceicao EP, Sim XYJ, et al. Minimising intra-hospital transmission of COVID-19: the role of social distancing. J Hosp Infect. 2020. https://doi.org/10.1016/j.jhin.2020.04.016.

2. Zhang J, Zhou L, Yang Y, et al. Therapeutic and triage strategies for 2019 novel coronavirus disease in fever clinics. Lancet Respir Med. 2020:8(3):e11-2. https://doi.org/10.1016/\$2213-2600(20)30071-0.

3. Huang C, Wang Y, Li X, et al. Clinical features of patients infected with 2019 novel coronavirus in Wuhan China. Lancet. 2020;395(10223):497506. https://doi.org/10.1016/S0140-6736(20)30183-5.

4. Ai T, Yang Z, Hou H, et al. Correlation of Chest CT and RT-PCR Testing in Coronavirus Disease 2019 (COVID-19) in China: a report of 1014 cases. Radiology. 2020. https://doi.org/10.1148/radiol.2020200642.

5. Tahamtan A, Ardebili A. Real-time RT-PCR in COVID-19 detection: issues affecting the results. Expert Rev Mol Diagn. 2020. https://doi.org/10.1080/ 14737159.2020 .1757437$.

6. Wolfel R, Corman VM, Guggemos W, et al. Virological assessment of hospitalized patients with COVID-2019. Nature. 2020. https://doi.org/10.1038/ s41586-020-2196-X

7. Guo L, Ren L, Yang $S$, et al. Profiling early humoral response to diagnose novel coronavirus disease (COVID-19). Clin Infect Dis. 2020. https://doi. org/10.1093/cid/ciaa310.

8. Feng H, Liu Y, LV M, et al. A case report of COVID-19 with false negative RT-PCR test: necessity of chest CT. Jpn J Radiol. 2020;38(5):409-10. https://doi.org/10.1007/s11604-020-00967-9.

9. Lippi G, Simundic AM, Plebani M. Potential preanalytical and analytical vulnerabilities in the laboratory diagnosis of coronavirus disease 2019 (COVID-19). Clin Chem Lab Med. 2020. https://doi.org/10.1515/ cclm-2020-0285.

10. Qu J, Wu C, Li X, et al. Profile of IgG and IgM antibodies against severe acute respiratory syndrome coronavirus 2 (SARS-CoV-2). Clin Infect Dis. 2020. https://doi.org/10.1093/cid/ciaa489.
11. Charlson ME, Pompei P, Ales KL, et al. A new method of classifying prognostic comorbidity in longitudinal studies: development and validation. J Chronic Dis. 1987;40(5):373-83. https://doi.org/10.1016/0021-9681(87) 90171-8.

12. Tobias A. Evaluation of the lockdowns for the SARS-CoV-2 epidemic in Italy and Spain after one month follow up. Sci Total Environ. 2020;725:138539. https://doi.org/10.1016/j.scitotenv.2020.138539.

13. Crobach MJ, Dekkers OM, Wilcox MH, et al. European Society of Clinical Microbiology and Infectious Diseases (ESCMID): data review and recommendations for diagnosing Clostridium difficile-infection (CDI). Clin Microbiol Infect. 2009;15(12):1053-66. https://doi.org/10.1111/j.14690691.2009.03098.x

14. Chen J, Qi T, Liu L, et al. Clinical progression of patients with COVID-19 in Shanghai China. J Infect. 2020;80(5):e1-6. https://doi.org/10.1016/j.jinf. 2020.03.004.

15. Fourati S, Hue S, Pawlotsky JM, et al. SARS-CoV-2 viral loads and serum IgA/lgG immune responses in critically ill COVID-19 patients. Intensive Care Med. 2020;46(9):1781-3. https://doi.org/10.1007/ s00134-020-06157-5.

16. Sun B, Feng Y, Mo X, et al. Kinetics of SARS-CoV-2 specific IgM and lgG responses in COVID-19 patients. Emerg Microbes Infect. 2020;9(1):940-8. https://doi.org/10.1080/22221751.2020.1762515.

17. Shiley KT, Van Deerlin VM, Miller WT Jr. Chest CT features of communityacquired respiratory viral infections in adult inpatients with lower respiratory tract infections. J Thorac Imaging. 2010;25(1):68-75. https://doi.org/ 10.1097/RTI.0b013e3181b0ba8b.

\section{Publisher's Note}

Springer Nature remains neutral with regard to jurisdictional claims in published maps and institutional affiliations.
Ready to submit your research? Choose BMC and benefit from:

- fast, convenient online submission

- thorough peer review by experienced researchers in your field

- rapid publication on acceptance

- support for research data, including large and complex data types

- gold Open Access which fosters wider collaboration and increased citations

- maximum visibility for your research: over $100 \mathrm{M}$ website views per year

At BMC, research is always in progress.

Learn more biomedcentral.com/submissions 